\section{Mel 09}

G GANGLIOSIDE: A MARKER FOR THE DIAGNOSIS AND TREATMENT OF NEUROECTODERMAL TUMORS?

H. Dippold, A. Knuth, H.P. Dienes, K.H. Meyer zum Büschenfelde

Flonoctona 7 antibody (mAb) $\mathrm{R}-24$, recognizing ganglioside $G_{D 3}$ on neuroectodermal tumors, shows several functional properties in vitro: inhibition of cell growth, complement fixation and mediation of antibody dependent cellular cytotoxicity (ADCC). The tissue specificity of this mAb R-24 was studied on 190 cryopreserved, unfixed human tissue sections by indirect immunoperoxidase staining. The specificity for neuroectodermal tumors was striking. Ganglioside $G_{D 3}$ was detected in all 21 tissue sections of 21 patients with primary melanoma and in 21737 probes of 24 patients with metastatic malignant melanoma. The majority of tumor cells in the samples of primary malignant melanoma expressed $G_{D 3}$ : however, GD3 expression was more heterogeneous in samples of metastatic lesions even in different metastases of the same patient. Of 18 nevi, 16 reacted with monoclonal antibody R-24, while melanocytes in the basal Tayer of normal sk in stained onty weakly and irregularly. Nane of the 32 normal and 12 fetal human tissue types were R-24 positive, but a strong cytoplasmic staining was observed with single cells in the dermis and in the interstitial tissue of the gastrointestinal tract, in the interlobular septa of the thymus, and in other distinct locations. Only two malignant carcinoid tumors of 38 nonmelanomatous tumors tested reacted with monoclonal antibody $\mathrm{R}-24$. The presented findings are particularly relevant in view of the clinical application of this mab in vivo. Local inflammatory response at the tumor site and tumor remissions have been observed after application of purified mAb R-24 in a percentage of patients. Side effects ( $s k$ in rash) have been minimal with this specific antibody.

New monoclonal ganglioside antibodies have been generated to cover the complete antigenic phenotype of neuroectodermal tumors.

I.Med.K1 inik, Joh.Gutenberg Universität, 6500 Mainz W.Germ.

\section{Mel 10}

COMPARISON OF THE IN SHORT-TERM METABOLIC ASSAY AND IN VIVO TREATMENT OF HUMAN MELANOMA XENOGRAFTS H. - J. Ochel, Glatte. P., Schmidt, C.G. and Osieka, R*. Histology and stage are major prognostic factors for the treatment of neoplastic disease but tumors with similar histology may respond heterogeneously to antineoplastic drug treatment. For the short-term metabolic assay extensive claims have been made as to its general applicability as a predictive test, although only two drugs are used to indicate sensitivity or resistance. This contrasts sharply with findings of other investigators who describe unique chemosensitivity patterns in xenografted human tumors $(1)$. We have exposed 3 human mela noma xenografts to alkylating agents and adriamycin and compared tumor growth curves to results from short-term assays performed on these xenografts. 'Str' is about 100 fold more sensitive to dacarbazine than 'GrII' and may be cured by intermediate doses of methyl-CCNu. Adriamycin is anly very weakly effective against this tumor, and an activated form of cyclophosphamide, mafosfamide (ASTA Z 7557). induces some growth delay. Methyl-CCNU, mafosfamide, and adriamycin were totally ineffective against melanoma "JaI'. although the latter line is somewhat sensitive to dacarbazine.

Jaking a $50 \%$ inhibition of ${ }^{3}$ H-uridine for ${ }^{3}$-thymidine) incorporation as a cutoff-point, tumor line 'str" was correctly classified as sensitive to methyl-CCNU and mafosfamide but falsely as sensitive to adriamycin. All three drugs were falsely classified as effective for completely resistant melanoma 'GrII', and for line ' JaI' only resistance to methyl-CCNU was indicated correctly by the short-term assay. Therefore, the limitation of predictive testing to one or two key antineoplastic orugs is not warranted by the neterogeneity present even among human tumors of similar histiogenetic origin.

(1) Osieka, R: Primary and acquired resistance to antineoplastic chemotherapy. Cancer 54:1168-1174 (1984) * Supported by BMFT (PTB 8315)

Westdeutsches Tumorzentrum. Hufelandstrabe 55, 43 Essen 1
Mel 11

T-LYMPHOCYTOTOXICTTY IN MALIGNANT MELANOMA: INDUCTION WITH AUTOIOGOUS MUTAGENIZEO TUMOR CETI CIONTS A.Knuth, A.van Pe1*, K.-H.Meyer zum Bueschenfelde, and

Mutagen treatment of spontaneous non-inmunogenic mouse tumors induces variants that elicit a strong rejection response in syngeneic mice. The rejection response is due to so-called tum antigens recognized by cytolytic T-lymphocytes (CTL). After rejection of tum tumor
cell clones mice show a certain degree of resistanc celi clones, mice show a certain degree of resistance tumor cells $\left(\right.$ tum $^{+}$). Our study was to investigate if this phenomenon of inmune protection would have relevance in human cancer inmunotherapy. Melanoma celis from a 38 year old patient with metastatic melanoma were adapted to tissue culture (MZ-MEL-2), cloned and repeatedly mutagenized in vitro with mutagenic compounds After several intracutaneous immunizations with iethally irradiated mutagenized autologous tumor cell clones peripheral blood mononuclear cells (PBL) were cultured in vitro with these melanoma clones, expanded in IL-2 containing culture medium and tested for cytolytic activity and specificity in vitro. Strong CIL activity direct Fostein-Barr-virus transformed autologous B-lynphocyte Fostein-Barr-virus transformed autologous B-lymphocy
cultures, $K 562$ cells or allogeneic melanoma targets was seen. Mutagenized as well as non-mutagenized autologous melanama target cells were lysed. The specificity of non-cloned CIL was confinmed in competitive inhibition assays We are presently investigating, if CTL bulk cultures or CTI clones after coculture with different mutagenized autologous tumor cell clones will preferentially lyse a singl tumor cells ( tum $\left.^{+}\right)$. This would be the evidence of mutagen induced expression of a new antigen (tum ${ }^{-}$). The clinical course of this melanoma patient evolved remarkably well under continued vaccination with repeatedly mutagenized autologous melanoma cell The patient remains in complete remission for over twelve months. A correlation between the unexpectedy favorable clinical course and the occurrence of an anti-tumor CTI response is suggested.

I.Medizinische Klinik und Poliklinik, Johannes Gutenberg-Universi taet, Langenbeckstr. 1 , D-6500 Mainz, *Iudwig Inst. Cancer Résearch, Ave. Hippocrate 74, UCI 7459, B-1200 Bruxelles, Belgium

\section{Mel 12}

\section{A RANDOMIZED PHASE-III-STUDY ON DTIC, LEVAMISOLE, and PLACEBO AS ADJUVANT TREATMENT OF HIGH RISK STAGE I PRIMARY HELANOMA}

E. Macher, U.R.Kleeberg, F. Lejeune, Ph. Rümke, M. Prade, D. Thomas, S. Suciu for the EORTC Halignant Helanoma Coop. Group (18761) - This study was designed to assess the value of chemotherapy vs. immunotherapy in high risk melanoma after surgical removal of a primary malignant melanoma of the skin. Eligibility criteria included the following conditions: previously untreated, histologically confirmed, primary cutaneous malignant melanoma of Clark stage III, IV, V with a Breslow's thickness equal or exceeding 1,5 $\mathrm{mm}$, no evidence for $7 y$ mphnode metastases. Only melanoma of the extremities were submitted to elective Iymphnode dissection. Randomization for 3 arms was done between DTIC and immunotherapy plus placebo. DTIC (250mg/m² b.s./dayx5, q4w) was given for a total of 6 cycles. Levamisole or placebo was taken orally on 2 consecutive days every week for 2 years (150-250 mg tablets per day according to weight). Results: 235 patients were entered and 94 considered as ineligible. Subgroup analysis reveals that the 96 limb melanoma patients did better than the 172 head, neck, and trunk patients: 5 years DFI $70 \%$ vs. $45 \%$ and 5 years $S 75 \%$ vs. $50 \%$ resp. This highly significant difference may be due in part to the histological selection of node-negative patients for limb melanoma. 107 out of 276 eligible patients reported a progression, which was local in 19, regional in 47 and distant in 41 patients.

Toxicity has been acceptable but higher in the DTIC arm where more protocol violations occured.

This report indicates that neither DTIC for 6 months not Levamisole for 2 years had any effect on disease free interval (DFS) or survival (S) of patients with high risk primary melanoma.

Haemat.-onkol. Praxis Altonà, 2000 Hamburg 50, Germany Secretariat of the EORTC Malignant Me lanoma Coop. Group 